\title{
The IRIS Network of Excellence: Future Directions in Interactive Storytelling
}

\author{
Marc Cavazza, Ronan Champagnat, Riccardo Leonardi and the IRIS Consortium ${ }^{1}$ \\ School of Computing, University of Teesside, Middlesbrough, TS1 3BA, United Kingdom \\ m.o.cavazza@tees.ac.uk
}

\begin{abstract}
The IRIS Network of Excellence (NoE) started its work in January 2009. In this paper we highlight some new research directions developing within the network: one is revisiting narrative formalisation through the use of Linear Logic and the other is challenging the conventional framework of basing Interactive Storytelling on computer graphics to explore the content-based recombination of video sequences.
\end{abstract}

Keywords: Narrative Formalisms, Planning, Linear Logic, Video Summarization, Hidden Markov Models, Interactive Storytelling, Interactive Narrative.

\section{Interactive Storytelling: Where Are We Going?}

The IRIS (Integrating Research in Interactive Storytelling) Network of Excellence (NoE) started its work in January 2009, as a new EC-funded initiative (under FP7's Intelligent Content and Semantics). It brings together 10 partners most of which have been prominent actors of the Interactive Storytelling community since this Conference series started in 2001. The new structure for NoEs in FP7 makes them more focussed, but also smaller in size: as such, they can no longer aim at structuring an entire community, and their relationship to the community as a whole has to be revisited. IRIS' researchers cover most of traditional areas of Interactive Storytelling (IS). They also approach IS from different theoretical perspectives, and debates within the consortium can reflect, and perhaps progress, those taking place within the IS community at large. Finally, IRIS can also be a laboratory for new ideas not simply in progressing topics on which there is consensus in the community, but in identifying radically different directions, and questioning accepted wisdom. IRIS' partners are contributing to a broad range of topics as can be seen by the IRIS papers presented at this conference [1] [2] [3] [4] [5] [6] [7] [8]. This paper highlights some current activities of the NoE, with an emphasis on some of its most original aspects.

\footnotetext{
${ }^{1}$ See the Acknowledgements section for the list of NoE members.
} 


\section{Non-Standard Narrative Formalisms: the Linear Logic of Madame Bovary}

One of the major issues of IS is the development of formalisms supporting narratives' descriptions. Ideally these formalisms should also be computational or support a translation to traditional computational formalisms. Logical descriptions have not been a popular formalism since the early work of [9] who have resorted to logical description and logic programming. There is however a case for exploring nonstandard logics, in particular when they embed concepts of high relevance to storytelling, such as causality. Linear Logic is precisely one such formalism. Linear logic has been introduced by J.-Y. Girard [10] as a restriction of classical logic. Unlike classical logic, linear logic is not used to determine whether an assertion is true or not but rather the validity of how formulas are used (and then consumed) when proving an assertion. It has, also, been introduced as a logic of resources [11]. Finally, linear logic is well suited to derive a computational model to partially ordered problems with resource sharing.

Greimas [12] [13] was the first to develop narrative formalisms as an abstract formula to represent an action. He proposed to define an action as a transition from one state to another state where the subject gains or loses an object (conjunctive or disjunctive narrative program). Greimas proposed to analyse a story as a sequence of actions, and we have proposed to use linear logic as a computational model to "compute" the story and to perform an analysis on the possible stories. The underlying model will stand in three points: the states of character and of the narrative, the story actions, and the story itself. Our example narrative is drawn from Madame Bovary, which is also studied by other activities in IRIS. We will also base part of our model on the characters' feelings, following [14].

We will focus on two characters from the novel (Emma and Rodolphe) and consider that Emma's state is given by two sub-states: one for her general mood (depressed $\left(E_{d}\right)$, happy $\left(E_{h}\right)$, in love $\left(E_{a}\right)$ ) and one for her feelings towards Rodolphe (Emma not acknowledging her love for Rodolphe $\left(\mathrm{E}_{\mathrm{rR}}\right)$, Emma seduced by Rodolphe $\left(E_{s R}\right)$, Emma in love with Rodolphe $\left(E_{a R}\right)$ ). As for Rodolphe, he may want to seduce Emma $\left(R_{v}\right)$, be in love with her $\left(R_{a}\right)$, or see his feelings for her waning $\left(R_{w}\right)$. A state is modelled by an atom of linear logic.

We also model the novel's plot as a set of states: conversation while Emma and Rodolphe attend the comices agricoles (agricultural show) $\left(\mathrm{D}_{\mathrm{c}}\right)$; in the council room $\left(\mathrm{D}_{\mathrm{s}}\right)$; delay $(\mathrm{T})$; at the Bovary's home $\left(\mathrm{D}_{\mathrm{b}}\right)$; horseback riding $(\mathrm{P})$; conversation at Rodolphe's home $\left(\mathrm{D}_{\mathrm{r}}\right)$. Six actions have been considered through the story: going to the agricultural show $\left(\mathrm{A}_{1}\right)$; listening to the speech in the council room $\left(\mathrm{A}_{2}\right)$; waiting before meeting again $\left(A_{3}\right)$; Rodolphe visits Emma $\left(A_{4}\right)$; horseback riding $\left(A_{5}\right)$; Emma visits Rodolphe $\left(\mathrm{A}_{6}\right)$. Each action is modelled by a formula of linear logic with a linear implication. The atoms on the left side of the linear implication connector correspond to the states required to perform the action. The ones on the right side are the ones derived by the action. For instance the action "listening discourse at the council room" requires the narrative to have the state $\mathrm{D}_{\mathrm{s}}$ available as well as Rodolphe state $R_{v}$ and $E_{h}$ and $E_{s R}$ for Emma, and will produce the following states: Rodolphe in love with Emma $\left(\mathrm{R}_{\mathrm{a}}\right)$ and Emma happy $\left(\mathrm{E}_{\mathrm{h}}\right)$ and seduced by Rodolphe $\left(\mathrm{E}_{\mathrm{sR}}\right)$. 


$$
\begin{aligned}
& A_{1}: D_{c} \otimes R_{v} \otimes E_{h} \otimes E_{s_{R}} \multimap R_{v} \otimes E_{h} \otimes E_{s_{R}} \\
& A_{2}: D_{s} \otimes R_{v} \otimes E_{h} \otimes E_{s_{R}} \multimap R_{a} \otimes E_{h} \otimes E_{s_{R}} \\
& A_{3}: T \otimes E_{h} \otimes E_{s_{R}} \otimes R_{a} \multimap E_{r_{R}} \otimes E_{d} \otimes R_{a} \\
& A_{4}: D_{b} \otimes R_{a} \otimes E_{d} \otimes E_{r_{R}} \multimap R_{a} \otimes E_{d} \otimes E_{r_{R}} \\
& A_{5}: P \otimes E_{d} \otimes E_{r_{R}} \otimes R_{a} \multimap E_{a} \otimes E_{a_{R}} \otimes R_{a} \\
& A_{6}: D_{r} \otimes E_{a} \otimes E_{a_{R}} \otimes R_{a} \multimap R_{w} \otimes E_{a} \otimes E_{a_{R}}
\end{aligned}
$$

Fig 1. Representation of Actions in Linear Logic.

The story can thus be modelled by the following sequent:

$D_{c} \otimes D_{s} \otimes T \otimes D_{b} \otimes P \otimes D_{r} \otimes R_{v} \otimes E_{h} \otimes E_{s_{R}} \otimes A_{1} \otimes A_{2} \otimes A_{3} \otimes A_{4} \otimes A_{5} \otimes A_{6} \vdash R_{w} \otimes E_{a_{R}} \otimes E_{a}$

In which the formulas modelling actions have been replaced by their name $A_{x}$.

Proving the sequent consists in rewriting the sequent by making q substitution of the formulas in order to have an initial sequent. The proof tree of the story is given by Fig 2 (bottom-up reading). The linear implication corresponds to an action of the story, and this proof give a manner to order the actions of the story.

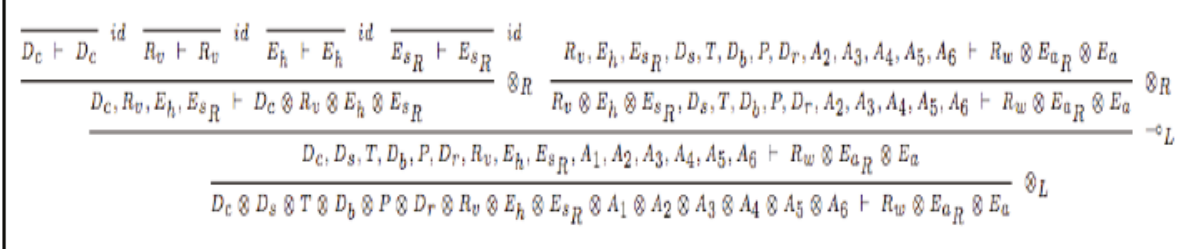

Fig 2. Proof of the Sequent Corresponding to an Excerpt of Madame Bovary.

The derived proof tree is very linear, which corresponds to the default course of action in Madame Bovary. However it can also provide alternative stories (in this specific case the actions that can be suppressed).

\section{Interactive Storytelling with Video?}

One of the basic dogmas of contemporary IS research has been that the generative nature of IS required similarly generative visual content, which could only be achieved through the use of computer graphics. The use of video was perceived as incompatible with the most recent AI-based narrative techniques and only compatible with various forms of branching techniques whose drawback was an explosion of prerecorded content. 


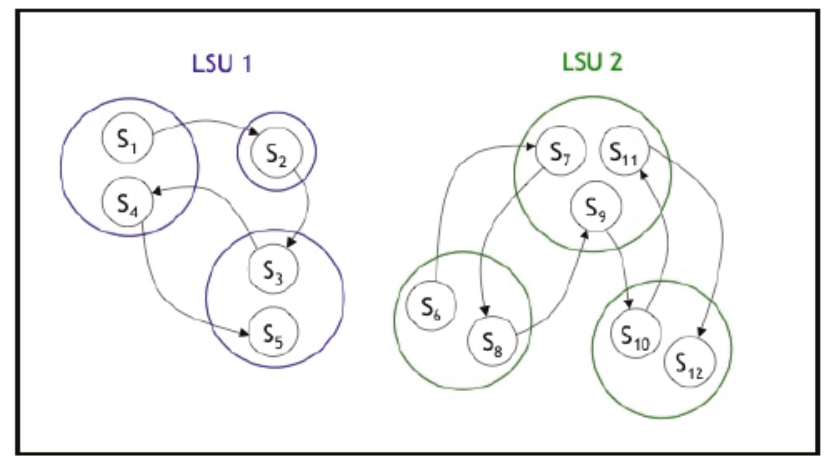

Fig 3. After the removal of cut-edges, each connected sub-graph of the STG represents a Logical Story Unit (LSU).

\subsection{Modelling feature films scenes using HMM}

Yeung et Yeo [15] shown that in a Scene Transition Graph (STG), after the removal of cut-edges, each connected subgraph well represents a Logical Story Unit (LSU), i.e., "a sequence of contiguous and interconnected shots sharing a common semantic thread", which is the best computable approximation to semantic scene [16]. In particular sub-graph nodes are clusters of visually similar and temporally close shots, while edges between nodes give the temporal flow inside the LSU, as shown in Fig 3 .

Starting from the STG representation, each LSU can be equivalently modelled by a Hidden Markov Model (HMM). This is a discrete state-space stochastic model which works quite well for temporally correlated data streams, and where the observations are a probabilistic function of a hidden state [16]. Such a modelling choice is supported by the following considerations (see [17]): i) Video structure can be described as a discrete state-space, where each state is a conveyed event (e.g., "man A talking") and each state-transition is given by a change of event; ii) The observations of events are stochastic since video segments seldom have identical raw features even if they represent the same event (e.g., more shots showing the same "man A talking" from slightly different angles); iii) The sequence of events is highly correlated in time, especially for scripted-content videos (movies, etc.) due to the presence of editing effects and typical shot patterns inside scenes to model higher level of abstraction concepts (i.e., dialogues, progressive scenes, etc.).

For the purpose proposed here, as HMM states representing events correspond to distinct clusters of visually similar shots; on the contrary state transition probability distribution capture the shot pattern structure of the LSU.

The original shots from which the HMM can be inferred constitute a possible observation set that has been produced by such stochastic model. 


\subsection{Using feature films' scenes HMM models for interactive "movietelling"}

Each LSU can be attached a specific semantics, which is conveyed/narrated through the underlying sequence of shots. It can be hypothesized that a mapping can be established between the semantic message and the inner states of the LSU representative HMM.

From each LSU, it can then be foreseen that innovative combinations and sequences of previously recorded shots can be constructed to convey variations of the original semantic message, thus paving the way for interactive movietelling.

HMM are used to instantiate possible alternative variations, by producing a likely chain of observations from already existing recorded material. This may be considered ambitious but the idea has already been proposed in the context of skim generation for feature films without significantly reducing the informativeness and enjoyability of the entertainment, as described in [18].

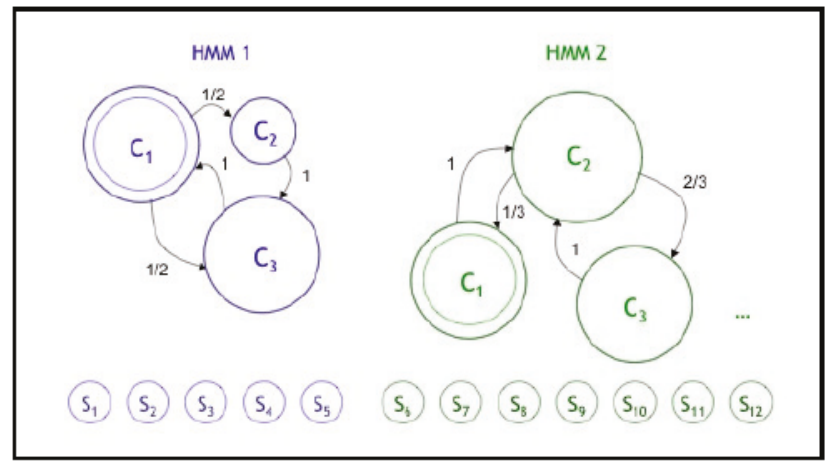

Fig. 4. LSUs of Fig 3 are equivalently modelled by HMMs.

\section{Conclusions}

IRIS' research programme aims at balancing progress on well-recognised key IS problems (such as authoring) and the ability to explore new avenues, such as videobased IS. The new directions we have presented are more connected to traditional IS problems that it may seem, and in their own way they lead to revisiting the notions of narrative action, causality, or the notion of scene and temporal unit.

To progress, the NoE also needs to envision various levels of integration. In the longer term, we should revisit key problems from an integrated perspective, such as the definition of narrative actions in-between narrative formalisms ("content") and AI formalisms ("form"). 
Acknowledgements. The IRIS Network of Excellence is funded by the European Commission (FP7-ICT-231824). IRIS members include Fred Charles, Julie Porteous (School of Computing, University of Teesside, UK); Stéphane Donikian, Marc Christie, Nicolas Pépin (IRISA/INRIA, FR); Ulrike Spierling, Steve Hoffmann (FH Erfurt, University of Applied Sciences, DE); Nicolas Szilas, Monica Axelrad, Urs Richle (TECFA, University of Geneva, CH); Peter Vorderer, Christian Roth, Ivar Vermeulen, Christoph Klimmt (CAMeRA, VU University Amsterdam, NL); Elisabeth André, Nikolaus Bee, Birgit Endrass, Katja Kurdyukova, Karin Leichtenstern (Institute of Computer Science, University of Augsburg, DE); Ronan Champagnat (University of La Rochelle, FR); Paolo Petta, Stefan Rank (Austrian Research Institute for Artificial Intelligence, AT); Patrick Olivier, Guy Schofield and Dan Jackson (Culture Lab, Newcastle University, UK), Riccardo Leonardi (University of Brescia, IT).

\section{References}

1. Endrass, B., Boegler, M., Bee, N. and André, E., 2009. What Would You Do in their Shoes? Experiencing Different Perspectives in an Interactive Drama for Multiple Users. In: Iurgel, I., Zagalo, N., Petta, P. (eds.): International Conference on Interactive Digital Storytelling, ICIDS, Guimarães; Springer Verlag (this volume).

2. Porteous, J. and Cavazza, M., 2009. Controlling Narrative Generation with Planning Trajectories: the Role of Constraints. In: Iurgel, I., Zagalo, N., Petta, P. (eds.): International Conference on Interactive Digital Storytelling, ICIDS, Guimarães; Springer Verlag (this volume).

3. Spierling, U. and Szilas, N., 2009. Authoring Issues Beyond Tools. In: Iurgel, I., Zagalo, N., Petta, P. (eds.): International Conference on Interactive Digital Storytelling, ICIDS, Guimarães; Springer Verlag (this volume).

4. Szilas, N. and Axelrad, M., 2009. To be or not to be: Towards Stateless Interactive Drama. In: Iurgel, I., Zagalo, N., Petta, P. (eds.): International Conference on Interactive Digital Storytelling, ICIDS, Guimarães; Springer Verlag (this volume).

5. Delmas, G., Champagnat, R. and Augeraud, M., 2009. From tabletop RPG to interactive storytelling: definition of a story manager for videogames. In: Iurgel, I., Zagalo, N., Petta, P. (eds.): International Conference on Interactive Digital Storytelling, ICIDS, Guimarães; Springer Verlag (this volume).

6. Kurdyukova, E., André, E. and Leichtenstern, K., 2009. Introducing Multiple Interaction Devices to Interactive Storytelling: Experiences from Practice. In: Iurgel, I., Zagalo, N., Petta, P. (eds.): International Conference on Interactive Digital Storytelling, ICIDS, Guimarães; Springer Verlag (this volume).

7. Roth, C., Vorderer, P. \& Klimmt, C., 2009: The motivational appeal of interactive storytelling: Towards a dimensional model of the user experience. In: Iurgel, I., Zagalo, N., Petta, P. (eds.): International Conference on Interactive Digital Storytelling, ICIDS, Guimarães; Springer Verlag (this volume).

8. Spierling, U., 2009. Conceiving Interactive Story Events. In: Iurgel, I., Zagalo, N., Petta, P. (eds.): International Conference on Interactive Digital Storytelling, ICIDS, Guimarães; Springer Verlag (this volume). 
9. Grasbon, D. and Braun, N., 2001. A Morphological Approach to Interactive Storytelling, Proceedings of Cast01, Living in Mixed Realities, Sankt Augustin, Germany, pp. 337-340.

10. Girard, J.-Y., 1987. Linear Logic, Theoretical Computer Science, London Mathematical 50:1, pp. 1-102.

11. Lafont, Y., 1993. Introduction to Linear Logic. Lecture notes from TEMPUS Summer School on Algebraic and Categorical Methods in Computer Science, Brno, Czech Republic.

12. Greimas, A.J., In: Sémantique structurale, Larousse, 1966 (in French).

13. Greimas, A.J., In: Du Sens, In: Essais sémiotiques. Le Seuil, 1970 (in French).

14. Pizzi, D., Charles, F., Lugrin J-L. and Cavazza, M., 2007. Interactive Storytelling with Literary Feelings. In Proceedings of ACII 2007, Lisbon, Portugal, September 2007, pp. 630-641.

15. Yeung M. M. and Yeo, B.-L., 1996. Time-constrained clustering for segmentation of video into story units. In Proeedings of ICPR'96, Vienna, Austria, Aug 1996, Vol. 7276(3), p. 375.

16. Rabiner, L. R., 1990. A tutorial on hidden Markov models and selected applications in speech recognition. In Readings in Speech Recognition, A. Waibel and K. Lee, Eds. Morgan Kaufmann Publishers, San Francisco, CA, pp. 267-296.

17. Xie, L., Xu, P., Chang, S., Divakaran, A., and Sun, H., 2004. Structure analysis of soccer video with domain knowledge and hidden Markov models. Pattern Recogn. Lett. 25, 7 (May. 2004), pp. 767-775.

18. Benini, S., Migliorati, P. and Leonardi, R., 2007. A statistical framework for video skimming based on logical story units and motion activity. In Proceedings of CBMI'07, Bordeaux, France, pp. 25-27. 